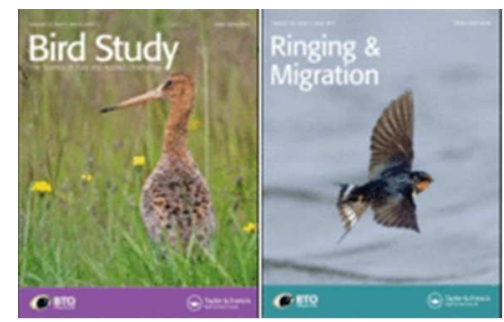

\title{
A first test of unattended, acoustic recorders for monitoring capercaillie (Tetrao urogallus L.) lekking activity
}

\begin{tabular}{|r|l|}
\hline Journal: & Bird Study/Ringing \& Migration \\
\hline Manuscript ID & BS-2017-066.R2 \\
\hline Danuscript Type: & Original Article \\
\hline Complete List of Authors: & $\begin{array}{l}\text { Abrahams, Carlos; Baker Consultants, ; Nottingham Trent University - } \\
\text { Clifton Campus, } \\
\text { Denny, Matthew; Denny Ecology, }\end{array}$ \\
\hline Keywords: & $\begin{array}{l}\text { bioacoustics, capercaillie, automated recording, bird display, lek, } \\
\text { monitoring }\end{array}$ \\
\hline
\end{tabular}

SCHOLARONE ${ }^{m}$

Manuscripts 


\section{A first test of unattended, acoustic recorders for monitoring capercaillie}

2 (Tetrao urogallus L.) lekking activity.

4 Carlos Abrahams ${ }^{1,2}$ \& Matthew J. H. Denny ${ }^{3}$

1. Corresponding Author: Baker Consultants, West Platform, Cromford Station,

6

(1)
01629593958

2. Corresponding Author: Nottingham Trent University, Clifton Campus, Nottingham, Nottingham Trent University, Clifton Campus, NG11 8NS

3. Denny Ecology, 17D Sturton Street, The Courtyard, Cambridge, CB1 2SN. 01223

13 Carlos Abrahams ORCID id: 0000-0003-0301-5585

15 Short title: Acoustic monitoring of capercaillie

16 Keywords: Bioacoustics, capercaillie, automated recording, bird display, lek

17 monitoring 


\section{Summary}

\section{Capsule}

21 Automated acoustic recording can be used as a valuable survey technique for capercaillie

22 leks, improving the quality and quantity of field data for this endangered bird species.

23 However, more development work and testing against traditional methods is needed to

24 establish optimal working practices.

\section{Aims}

26 The use of bioacoustics is a rapidly developing tool for ecological research, but still requires

27 testing across a variety of taxa and recording environments. This study aims to determine

28 whether capercaillie vocalizations can be recognized in lek recordings, whether this can be

29 automated using readily available software, and whether the number of calls resulting varies

30 with location, weather conditions, date and time of day.

\section{Methods}

32 Unattended recording devices and semi-automated call classification software were used to

33 record and analyse the display calls of capercaillie at three known lek sites in Scotland over a

34 two week period.

\section{Results}

36 Capercaillie calls were successfully and rapidly identified within a dataset that included the

37 vocalizations of other bird species and environmental noise. This demonstrates that calls can

38 be readily recognized to species level using a combination of unsupervised software and

39 manual analysis, and the number of such calls counted to gain an index of lek activity. The

40 number of calls varied by time and date, and by recorder/microphone location at the lek site,

41 and was related to weather conditions. This information can be used to better target future

42 acoustic monitoring and improve the quality of existing traditional lek surveys.

\section{Conclusion}


44 This study is a contribution to the development of bioacoustics as a practical and cost-

45 effective method for determining habitat occupancy and activity levels by a vocally

46 distinctive bird species. Following further testing alongside traditional counting methods, it

47 could offer a significant new approach towards more effective monitoring of local population

48 levels for capercaillie and other species of conservation concern.

49 


\section{Text}

\section{Introduction}

52 Western capercaillie (Tetrao urogallus L.) is a bird of high conservation concern in the UK, 53 and elsewhere in Europe, on account of its low population size and historical decline (Storch 54 2000; Eaton et al. 2015). Thought to have become extinct in Scotland in the mid to late $18^{\text {th }}$ 55 century, it was successfully reintroduced, but has declined again in the $20^{\text {th }}$ century. Whilst 56 the reasons for this decline are complex and not fully understood, research has shown that

57 low breeding success associated with climate change, and mortality resulting from adult birds

58 flying into forest fences, have contributed to the decline (Moss 2001; Ewing et al. 2012). The

59 Scottish capercaillie population has been subject to concerted conservation management

60 efforts over the past few decades, which appear to have stabilised the population at a

61 critically low level, but not increased it (Wilkinson 2017), rendering it susceptible to

62 extinction again in Britain (Moss 2001).

63

64 A range of methods have been used for capercaillie monitoring, including counts of

65 displaying males at leks (Picozzi et al. 1992; Summers et al. 2010) and genetic capture-

66 recapture techniques (Jacob et al. 2010) to assess population status. For national status

67 surveys in Scotland, line transects are conducted in winter (Ewing et al. 2012). However, the

68 species currently has a low population density and variable detectability relating to habitat

69 type, sex and temperature (Ewing et al. 2012). As a result, the 2009-10 national transect

70 survey only recorded an average of one capercaillie encounter per $22.2 \mathrm{~km}$ of transect. Whilst

71 there are good reasons for applying a winter transect count method for the national survey

72 (Ewing et al. 2012), the low encounter rates hinder the ability of this survey method to

73 sensitively track changes in the population at smaller temporal and spatial scales. 
75 Capercaillies have a polygonous mating system with an 'exploded' lek breeding system,

76 where males display over a dispersed area to indicate their breeding condition and quality

77 (Wegge et al. 2013). The leks occur in forest habitat, centre on a display ground covering an area of c. 0.30 hectares, and have mean numbers of male birds of between 0.5 and $20+$ per lek, dependent on the quality and amount of the surrounding old forest habitat (Hjorth 1970;

80 Picozzi et al. 1992; Angelstam 2004; Laiolo et al. 2011). Since 2002, capercaillies in

81 Scotland have been counted at lek sites each April, with a subset of 69 leks subject to

82 consistent monitoring effort. Between 2004 and 2010, the number of male birds at regularly

83 counted leks declined from 215 to 152 birds, a fall of 29.3\% (Ewing et al. 2012). This may

84 have been due to an overall population decline, or abandonment of traditional lek sites in

85 favour of new sites, or a combination of these processes. One of the advantages of acoustic

86 monitoring is the potential for wider spatial and temporal systematic sampling, facilitating the

87 identification of newly occupied lek sites.

89 The quality of data from traditional lek counts may be affected by differences in detection

90 probabilities between habitats or survey events (e.g. in ambient background noise), or

91 measurement and identification errors. Biases may occur in traditional bird count data, with

92 large inter- and intra-observer errors (Celis-Murillo et al. 2009; Simons et al. 2009) -

93 sometimes due to existing knowledge about the survey area (Hancock et al. 1999). For

94 capercaillie, the surveyed lek sites are often remote, experienced surveyors are few in

95 number, and the necessary timing and seasonal constraints on field survey methods raise

96 difficulties. As a result, the spatial and temporal coverage of capercaillie sites is currently

97 limited, leading to low confidence in the results from point counts. In addition, capercaillies

98 are known to be susceptible to human disturbance (Ewing et al. 2012), and regular

99 disturbance due to traditional counts has the potential to negatively affect populations. There 
100 is a clear need for improved monitoring techniques, especially at important sites, or locations

101 where management actions have been implemented, to determine site occupancy and finer

102 scale temporal and spatial trends. In this way, significant short-term population changes

103 could be identified more readily to alert conservationists to both acute problems and

104 management intervention success. The use of automated acoustic detection, alongside

105 existing survey methods, could reduce the recognised biases and act as a complementary

106 method to enable more accurate population estimates, but there are always going to be

107 logistical and cost implications undertaking both methods in parallel.

109 As an alternative or complement to existing techniques, we test here the use of unattended

110 sound recorders (often called 'passive' or 'autonomous' recorders) for monitoring

111 capercaillie leks. Recording of vocalizations has previously been used to monitor other bird

112 species, such as bitterns (Gilbert et al. 2002), corncrakes (Peake \& McGregor 2001) and

113 nightjars (Zwart et al. 2014). Unattended sound recording is especially applicable in

114 situations where populations are remote, sensitive to disturbance, or the species is cryptic, as

115 recorders can be deployed in the field for long periods of time with minimal surveyor

116 influence at the monitoring site. Hence, this method is potentially highly applicable for

117 capercaillie.

118

119 The displays of capercaillie males at lek sites commonly entail a sequence starting with

120 vocalizations from a tree perch, before moving to the ground to commute to the lek centre

121 and later adding visual signals to their continuing display songs (Wegge et al., 2013). The

122 typical full capercaillie display song (Figure 1) consists of a low frequency broadband rattle

123 between 1 and $5 \mathrm{kHz}$, then a deep pop, followed by a repeated scratchy sound between 2.5 
124 and $6.5 \mathrm{kHz}$. This sequence is described as "drum roll - cork pop - whetting" by Liaolo et al. 125 (2011).

126

127 As part of a monitoring programme, effective recording and recognition of capercaillie

128 vocalizations within large audio datasets could allow the occupancy of a site to be

129 determined, and an index of relative use to be developed (e.g. Briggs et al. 2012). It may also

130 be possible to assess the number of male birds at a lek from sound recordings. Laiolo et al.

131 (2011) found that capercaillie song rate (the number of songs per minute from an individual

132 bird) is significantly associated with the number of displaying males. This is likely to be as a

133 signal of intimidation, as the birds attending the lek stimulate each other by increasing their

134 vocal display. Therefore, song rate, recorded using automated bioacoustic techniques, could

135 be used as a proxy for lek counts undertaken by traditional methods.

137 This study sets out to determine whether capercaillie vocalizations can be recognized in

138 recordings to species level, and whether this recognition can be automated and calls counted

139 using readily available software. The results are then used to determine how the number of

140 calls varies according to location, weather conditions, date and time of day.

\section{$142 \quad$ Materials and methods}

143 Four Wildlife Acoustics (www.wildlifeacoustics.com) SM2 acoustic recorders were placed at

144 known capercaillie lek sites near Aviemore, Scotland $\left(57.19^{\circ} \mathrm{N}, 3.82^{\circ} \mathrm{W}\right)$ in April 2016.

145 Each recorder was programmed to record in stereo, with one Wildlife Acoustics SMX-II

146 omnidirectional microphone (left channel, 0) mounted on the recording unit, and another

147 (right channel, 1) at the end of a 50m extension cable. The recorder and cabled microphones

148 were both attached to trees at approximately $1.5 \mathrm{~m}$ off the ground, and oriented horizontally in 
149 opposite directions N-S or E-W. The microphone and recorder were both placed in the

150 vicinity of the lek centre as indicated by a surveyor familiar with the sites and the normal lek

151 count hide locations. GPS coordinates were taken for all recorder and microphone locations.

152 The four recording devices were placed at three lek sites, each separated by a distance of

153 kilometres. At one lek site, two recorders (9333 and 9898) were placed together, with the

154 four microphones mounted on the recorders and associated cables forming the corners of an

155 approximate $50 \times 50 \mathrm{~m}$ square. The reason for doing this was the fact that previous count

156 surveys and checks for field signs had been unable to accurately define the location of the lek

157 at the site.

158

159 The recorders were programmed to record between 04:00-10:00 every day, starting at 04:00

16023 April 2016 and ending at 10:00 on 6 May 2016. Recording was limited to these times

161 based on standard lek count practice and surveyor advice (Haysom 2013; S. West pers.

162 comm.), whilst saving the limited battery life and data storage capacity. Sunrise time at the

163 start of the survey period was at $05: 46$, getting earlier to $05: 14$ at the end of the survey.

164 During each survey day, the recorders created a series of 10 minute duration full-spectrum

165 data files in Waveform Audio File (.wav) format, recording at a sampling rate of 24,000 $\mathrm{Hz}$

166 and 16 bits per sample (see Technical Appendix).

168 The survey provided a data set covering 14 days ( 84 hours) at each of the four recorders, with

169 the data from each recorder comprising 505 stereo files (total 2,020). The final day of

170 recording (04:00-10:00 6 May) was used to produce a set of training data for developing an

171 automated call recognizer in the software. The remaining 13 days were retained for analysis

172 purposes.

173 
174 Data were analysed using Kaleidoscope Pro 4.0.0 software (Wildlife Acoustics 2016), using

175 its 'cluster analysis' method. This process searches for repeated phrases in the recordings

176 (e.g. the song of a particular bird species) and groups these into a number of clusters based on

177 their similarity. It provides a numerical score to quantify the 'distance' of each individual

178 vocalization phrase from the centre of the cluster (low scores being better matches with this

179 average). According to the software protocol, a preliminary analysis was conducted on the

180 training data to scan and cluster recordings. The clustering process identified individual

181 'phrase segments' within the training data, each of these being a mono recording (from either

182 the right or left channel), $>2$ and $<7$ seconds in duration (the typical song length of

183 capercaillie), comprising a sequence of 'syllables' occurring close enough together in time

184 such that the defined 'maximum inter-syllable gap' of 1 second is not exceeded. All the

185 phrase segments from the training data were individually reviewed and manually identified as

186 either capercaillie calls or other sounds, by viewing the sonogram and listening to playback.

187 In addition, the performance of the clustering process was assessed by comparing clustered

188 data to a stratified sample of the original recordings. Each phrase segment selected by

189 clustering could include vocalizations by more than one bird species, if these were singing

190 simultaneously within the frequency band, but they were assigned as capercaillie if calls from

191 this species were included. From this manual review, the cluster with the highest proportion

192 of capercaillie phrases from the training data was identified, and this cluster was then used as

193 a recognizer to identify matching capercaillie phrases within the 13 day sequence of analysis

194 data, using the same analysis parameters as used for the training data.

195

196 To assess the effectiveness of the classification process, all the phrase segments identified in

197 the analysis data as 'capercaillie' matches were manually checked by viewing the sonogram

198 and listening to playback. This allowed the proportion of false positive matches to be 
199 assessed. To identify the proportion of false negatives, a random selection of 500 (4\%) 'non-

200 capercaillie' phrase segments from the analysis data was also manually checked.

201

202 As environmental context for the acoustic data, weather data for the Met Office MIDAS

203 station at Aviemore was accessed through BADC (badc.nerc.ac.uk/cgi-bin/midas_stations/

204 station_details.cgi.py?id=113\&db=midas_stations), and DATA.GOV.UK (using the

205 Aviemore weather station codes DCNN 0585 and RAIN 817692). Daily rain data for

206 Northern Scotland was also accessed from Hadley UKP (www.metoffice.gov.uk/hadobs/

207 hadukp/data/download.html).

208

209 Statistical tests were carried out using R and R Studio software (R Core Team 2015; RStudio

210 Team 2015).

211

$212 \underline{\text { Results }}$

213 The first stage of analysis used clustering to identify and group similar vocalizations within

214 the single day of training data. This identified 5401 individual phrase segments, produced by

215 a variety of bird species, grouped into 10 clusters. The total duration of these phrase segments

216 amounted to 4.88 hours, out of a total recording time of 48 hours (4 recorders $\times 6$ hours $\times 2$

217 channels). All 5401 training data phrase segments were manually reviewed (taking less than

218 eight hours), with 258 segments (5\%) identified as having capercaillie calls, and 5143

219 segments without capercaillie (Table 1). Of the 5401 phrase segments, 80 were assigned to

220 Cluster 09, in which $52(65 \%)$ were manually confirmed to contain capercaillie calls (the

221 highest proportion of capercaillie calls of any cluster). The remaining 206 phrase segments

222 that included capercaillie vocalizations (often overlapping calls from other species) were

223 spread through the remaining clusters. Most of these were in Cluster 08, which had 
224 capercaillie vocalizations in $20.1 \%$ of its phrase segments, while all remaining clusters had 225 less than $5 \%$ of phrase segments being positive for capercaillie. Hence, clustering of the 226 training data at this initial stage provided a single main capercaillie cluster which picked out $22752(20 \%)$ of 258 capercaillie phrase segments manually identified from the dataset. The 228 check back of clustered data against the original recordings showed that the clustering 229 performed well according to the set parameters. The clustering correctly identified the 230 presence or absence of capercaillie in the 10 -minute wav files $75 \%$ of the time, with false 231 positives (calls incorrectly assigned to Cluster 9) in $8 \%$ of cases, and false negatives (calls 232 missed or assigned to another cluster) in $17 \%$ of cases. This manual review also indicated that there were a number of short capercaillie sequences or individual spaced calls present, that were outside the parameters of the clustering process due to their limited duration (often being less than 1 second).

Using Cluster 09 to identify similar capercaillie recordings, the remaining 13 day sequence of analysis data was processed to determine whether capercaillie phrases could be effectively identified within the recorded dataset. A total of 13,626 phrase segments were produced from the analysis data (Table 1), of which 907 (6.7\%) were assigned as a match to the Cluster 09 capercaillie data. These were all manually checked and 758 of the 907 (83.6\%) were confirmed as capercaillie, with $149(16.4 \%)$ false positive matches. To identify the proportion of false negatives, a random selection of 500 phrase segments (4\%) out of the remaining

24412,719 were manually checked. Of these, 55 phrases (11\%) were confirmed as including capercaillie vocalizations and hence being false negatives. The greatest proportion of these were in Cluster 08, which had 29\% false negatives, and Cluster 01, which had 13\%. The remaining clusters $02-07$ all had a false negative proportion of $<10 \%$. Hence, overall there were estimated to be $1399(0.11$ x 12,719) phrase segments containing capercaillie calls in 
249 the analysis dataset which were not discovered. This equates to the supervised clustering

250 successfully identifying $83.6 \%$ of capercaillie vocalizations in Cluster 09 , and correctly

251 extracting $35 \%$ of all capercaillie phrase segments. These findings mean that the limited

252 number of false positives in Cluster 09 could be manually screened quickly, with a low rate

253 of false negatives scattered through the other clusters - these often being low 'quality' phrase

254 segments with single calls or poorly recorded, and therefore difficult and time-consuming to

255 identify manually.

256

257 The dataset of 758 capercaillie phrase segments identified by the cluster process and manual

258 confirmation was used for further analysis. The spectrograms were first analysed to ascertain

259 the characteristics of the recorded calls. Within the dataset, the vocalizations had a mean

260 frequency of $3083 \mathrm{~Hz}$, within a general range of 2000-4000 Hz (Figure 2). Some variation

261 was found between the data from different locations, with means between $2874 \mathrm{~Hz}$ at

262 recorder 9558 and $3234 \mathrm{~Hz}$ at 9333 . A median duration of 4.512 seconds was found for the

263 identified phrase segments, with a minimum of 2 seconds and a maximum of 6.94 seconds

264 (as constrained by the software settings).

265

266 The differences in the total number of recorded phrase segments (from all species), and those

267 of capercaillie, were investigated across different recorder locations and between left and

268 right stereo channels. The numbers of all of these varied widely between recorder locations,

269 with almost no vocal activity recorded at 9333, moderate levels at 9558 and highest activity

270 at 9898 and 9573 (Table 2). As context, the number of males recorded during lek counts at

271 these sites in the same season (but not concurrently with recording), were three birds at

272 9333/9898, five at 9573 and 7 at 9558 (S. West, pers. comm.). A great deal of variation was

273 found between the two stereo channels on each recorder, with all locations recording many 
274

275

276

277

278

279

280

281

282

283

284

285

more calls on one channel than the other. Review of the capercaillie call data revealed very few instances ( $\mathrm{n}=8, \mathrm{c} .1 \%)$ where near-simultaneous calls were recorded on both left and right channels, i.e. from the same bird being recorded simultaneously on both channels. Hence large differences were found between data from microphones located 50 metres apart. In addition, recorders 9333 and 9898 were both placed in the vicinity of a single lek site and recorded widely differing numbers of vocalizations. A possible reason for this is discussed below.

The number of calls recorded per day was investigated to determine whether there was any trend across the survey (and lekking) period. The overall levels of capercaillie vocal activity, pooled across all recorder locations, varied day-to-day between 1-191 phrase segments, but were highest at the start ( $23^{\text {rd }}$ April) of the survey and declined (with daily variations) throughout the rest of the period (Figure 3). This is likely to reflect a true decline in lekking activity, as the survey was undertaken at the tail end of the main lekking season. The highest daily total of phrase segments at a single recorder was a maximum of 146 at recorder $9898-$ this being more than half of all segments recorded at that location, recorded in a single day.

Prior to the study, an early morning peak in vocal activity was expected, with units set to record between 04:00-10:00. This assumption was found to be correct, with our data clearly indicating that the highest levels of call activity were recorded in the 2 hour period around sunrise (Figure 4), with a median time for all calls of 36 minutes before sunrise. There are significant differences between the recorder locations though (Kruskal-Wallis chi-squared = 289.13, df $=3$, p-value $<0.01$ ), with unit 9573 being significantly earlier than the other three locations. 
299 If the morning peak in activity is related to sunrise time (i.e. light levels), then we would

300 expect this to get earlier through the survey period as day length changes. This relationship

301 between peak vocalization time and sunrise appears to be demonstrated in Figure 5, where in

302 addition, the high level of calls around 04:00 am, the start of the recording session, are

303 indicated.

305 Relationships between the total number of vocalizations per day with three weather

306 parameters were tested using Spearman's rank correlation (Table 3). A significant negative

307 correlation $(\mathrm{p}<0.05)$ was found with windspeed (Figure 6$)$, but there was no clear relationship

308 with temperature or amounts of rainfall.

\section{Discussion}

311 Our results confirm that automated passive acoustic recorders can effectively be used to

312 detect and record capercaillie vocalization activity in the field. This study has also shown that

313 semi-automated call analysis can rapidly identify individual vocalization phrases for a target

314 species, with call classification having an accuracy of $>80 \%$ accuracy and correctly

315 extracting $35 \%$ of all capercaillie calls (most of those not extracted being of poor-quality) -

316 and only producing $16 \%$ false positives. The clustering process applied here is a different

317 approach to the use of pre-constructed species-specific recognisers used in many other

318 studies (Brandes 2008; Bardeli et al. 2010; Oppel et al. 2014). It is primarily intended to be a

319 human-supervised process which organises sound data into call-type groups to allow rapid

320 manual review and labelling. With the appropriate manual checks, including identification of

321 false negative and positive classifications, it was very successful in correctly identifying

322 capercaillie vocalizations in the analysis dataset, even when based on a small single set of

323 training data - albeit with a relatively high omission error (64.9\%). Although the clustering 
324 process used here, based on a limited number of individuals, was suitable for identifying

325 birds at the study sites, it is expected that improved rates of detection, with fewer false

326 positives and negatives, could be achieved in future studies with a larger training dataset

327 (Digby et al. 2013). In addition, it is worth noting that our method did not attempt to

328 exhaustively identify every capercaillie vocalization in the recorded dataset. The clustering

329 approach allowed a user-determined set of search parameters to be applied to the data, with

330 vocalizations that matched the settings being selected as phrase segments. As a result, it is

331 accepted that vocalizations not matching these criteria (e.g. short individual calls) would not

332 have been identified, and the capercaillie phrase segments used in our analysis are a reduced

333 subset of the overall recorded activity. However, the defined criteria used in the clustering

334 ensures that vocalizations of the same type and quality are being compared between different

335 days and detector locations, allowing a coherent analysis of the call data. This rapid analysis

336 method, with low levels of false positives, is particularly suited to ascertaining the presence

337 of capercaillies at a site, which could be a very useful tool for a species with low densities

338 and fluctuating lek site occupancy.

340 The numbers of calls recorded varied widely between recorder locations and also between

341 left and right channels on the same recorder. The former could indicate differences in the

342 levels of vocal activity between different lek sites, while the latter indicates that capercaillie

343 calls do not travel well over distance, i.e. detectability is limited at distances $=>50$ metres.

344 This is similar to detection ranges found in other bioacoustic studies of forest birds, e.g.

345 Venier et al. (2012) and Sedláček et al. (2015). Using the same type of recorders and

346 microphones, Turgeon et al. (2017) found bird call detection radii of between 13-203 metres,

347 dependent on the species, background noise levels and microphone condition. For

348 comparison, the spacing between individual capercaillies at leks has been recorded as 64-212 
349 metres (with interactions between males sometimes occurring at less than 10 metres), and

350 calls from this species can generally be heard at a distance up to approximately 200 metres by

351 the human ear (Hjorth 1970; Wegge et al. 2013). This relationship between detection

352 distances and bird density clearly raises the issue of detectability during surveys, for both

353 human point counts and automated recording equipment (Yip et al., 2017). This indicates

354 that, for bioacoustics methods, careful thought needs to be given to the number, layout, and

355 response of recorders and microphones, as well as the characteristics of the recording

356 environment. In addition, when recording and analyzing sound files, the appropriate audio

357 settings, such as gain, sample rate, and the use of high and low pass filters should be

358 considered. The development of good practice guidance for this should be prioritized to

359 ensure repeatable results from any future monitoring programme (Eyre et al. 2014; Pocock et

360 al. 2015), and further research should focus on elucidating the optimum number of

361 microphones, and distance between them, at a lek site.

363 In this study, the pair of recorders 9333 and 9898 were located either side of a wide

364 electricity pylon wayleave through the forest, with the lek site thought possibly to be present

365 within the open wayleave habitat between. However, the recorder on the northern side of the

366 wayleave (9898) recorded 265 capercaillie phrases, compared to only 4 on the south side

367 (9333). This is likely to indicate that the lek site was actually present within the forest to the

368 north of both recorders, and audible sound data was only picked up at the closest set of

369 equipment.

370

371 Differences were found in median call timings between locations, with recorder location

3729573 being significantly earlier in the day compared to other locations. This could perhaps be

373 due to habitat differences, such as forest structure, aspect or altitude. For example, 9573 was 
374 the lowest of all four sites at $255 \mathrm{~m}$ altitude and in relatively open forest habitat, while the

375 rest were at 325-375 $\mathrm{m}$ in denser plantations. Further exploration of how the environment

376 might affect capercaillie lekking behaviour in this way would be worthwhile (Angelstam

377 2004; Laiolo et al. 2011).

378

379 Lek monitoring at the local scale, rather than winter transect counts which are subject to low

380 encounter rates (Ewing et al. 2012), should be seen as an important method of monitoring the

381 effects of management and alert practitioners to local population changes. As discussed

382 already, there are significant limitations to traditional manual lek counts, and this automated

383 acoustic approach provides a promising alternative or complement. Within our study, large

384 differences were found between the number of capercaillie vocalizations recorded at each of

385 the three locations. This may partly be due to the precise location of the recorders in relation

386 to the lekking birds, given the range detectability issue discussed above (which is also likely

387 to affect human observers), but could also be a true reflection of bird numbers and activity

388 levels at each site. We anticipate that the level of call data recorded using our methods should

389 be indicative of population size and lekking activity, but comparison with human observer

390 counts has not been attempted in this study, due to the limited number of leks covered and the

391 lack of synchronous count data. Further work is clearly required in this area, but studies have

392 shown that recorded calling rates are positively relate to lek size in white-bearded

393 manakin Manacus manacus (Cestari et al. 2016) and white-bellied emerald Amaziliu candida

394 (Atwood et al. 1991); and to nest density at Cory's shearwater Calonectris borealis breeding

395 sites (Oppel et al. 2014). These findings indicate that acoustic monitoring may be useful to

396 document relative changes in local bird populations over time. In particular, the day-to-day

397 variation we recorded in call activity at each site over the survey period (summarised in 
398 Figure 3) must sound a note of caution to reliance on capercaillie population data from single

399 visit lek counts.

400

401 Haysom (2013) recommends that capercaillie lek surveys in Scotland should take place

402 during the peak period of mid-April to early May (with variation according to spring

403 temperatures). The call activity we recorded was highest at the start of the survey period (23

404 April) and declined through the survey period. Hence, this indicates that earlier activity might

405 have been missed in this study, Further unattended acoustic research of capercaillie leks

406 should aim to test whether there is activity prior to mid-April, to understand whether the

407 recommended seasonal parameters of traditional lek surveys need to be adjusted.

408

409 The peak of highest levels of call activity, across all recorders, occurred at 36 minutes before

410 sunrise. The standard guidance by Haysom (2013) recommends that leks should ideally be

411 counted from 04:00 to 06:00 hours. However, relatively high levels of call activity were

412 recorded at the start of our daily survey period (4-10 am), so for future studies, an earlier start

413 to survey is recommended, e.g. 2-3 hours before sunrise (c. 2:30-3:30am).

414

415 The number of recorded vocalizations decreased with increasing wind speed. This could be

416 due to: (i) reduced calling (and lekking) activity in adverse weather conditions, (ii) reduced

417 detectability of calls in high winds, or (iii) increased masking by background noise in high

418 winds (Digby et al. 2013). There is anecdotal recognition of the effects of environmental

419 parameters - weather and altitude - on call activity from human observers at lek counts. The

420 impacts of this on results could do with further investigation to allow the quality of count

421 data to be assessed against weather conditions, with weather factors (if recorded) being

422 modelled into data analysis and therefore removing this source of variation. It would be more 
423 practicable to achieve this with the long datasets possible from automated recording, than

424 those provided by the limited resource of human surveyors (Oppel et al. 2014).

425

426 In conclusion, this study has shown that capercaillie can be effectively recorded in the field

427 using automated passive acoustic methods. The equipment necessary to do this is simple and

428 readily available, and enormous progress in signal processing and pattern recognition in

429 recent years has made it possible to incorporate automated methods into the detection of

430 vocalisations (Bardeli et al. 2010). As a result, there is a clear opportunity for acoustic

431 monitoring of this species over extended periods, with rapid analysis of the recorded

432 vocalizations. The time and cost savings of this approach over manually reviewing all of the

433 sound data are significant. In this study, a total of 56 'days' of recording was completed with

434 two days of fieldwork, and one-two days of call analysis. This is not uncommon - Digby et

435 al. (2013) assessed that autonomous recorder methods required $<3 \%$ of the time needed for a

436 comparable traditional field survey.

438 The continuing vulnerability of the Scottish population of capercaillie makes regular and

439 consistent monitoring a priority. The use of acoustic techniques could eliminate or minimize

440 observer biases, reduce disturbance caused by surveyors, and provide standardized field data

441 that can be permanently archived. It could also help resolve problems associated with

442 surveying in pre-dawn darkness, hard to access survey sites and with the limited availability

443 of expert field observers (Hobson et al. 2002; Celis-Murillo et al. 2009; Zwart et al. 2014).

444 Acoustic recording methods could allow for cost-effective lek occupancy checks of suitable,

445 but previously unmonitored or unoccupied, areas, which would be unfeasible using manual

446 lek surveys. Acoustic data may also be useful in testing when (in terms of weather conditions,

447 season and time of day) manual monitoring would be most effective, and could help gauge 
448 the accuracy of point counts. As a result, it is a developing tool that could potentially have

449 great application and significance, offering to fill a methodological gap especially for the

450 census of cryptic taxa such as capercaillie (Dawson \& Efford 2009; Bardeli et al. 2010;

451 Laiolo 2010; Zwart et al. 2014).

452

453 The next step in the development of bioacoustics for birds should be in the establishment of

454 recognized survey protocols and statistical approaches to be employed by practitioners such

455 as conservation professionals and ecological consultants (Marques et al. 2013), to set out

456 good practice and allow greater comparability between studies of different species and at

457 different locations. This will require testing and work to compare traditional versus acoustic

458 methods - probably developing an improved approach which combines the two into an

459 integrated system. For capercaillie, the obvious first step is to correlate lek count numbers

460 against the numbers of calls recorded during the same survey event, or better, over a longer

461 survey period surrounding a number of repeated counts at each lek.

462 


\section{Acknowledgements}

464 Many thanks to RSPB staff, Sarah West and Gareth Marshall, for enabling this study and 465 assisting with deployment of recording equipment, and to the landowners who allowed access 466 to their sites.

468 Technical Appendix - Recording and Analysis Settings

469 Wildlife Acoustics Songmeter SM2 recorders were used. Recording was constant during the 470 set times, without triggers being set. No high or low pass filters were used, and a gain setting 471 of $+48 \mathrm{~dB}$ was applied. The SMX-II microphones used have a typical sensitivity of -40 to -43 $472 \mathrm{dBV} /$ pa and frequency response of 20-20000 Hz (Ehnes \& Foote, 2015; Turgeon et al., 473 2017).

475 For call analysis with Kaleidoscope Pro the following analysis parameters were used: Daily 476 subdirectories created; Files split to 60s max duration; Split channels; Signal of interest 15004774000 Hz; Duration 2-6s; Maximum inter-syllable gap 1s; Max distance from cluster center to 478 include outputs in cluster.csv $=1.0 ;$ FFT window $=5.33 \mathrm{~ms}$; Max states $=12$; Max distance to 479 cluster centre for building clusters $=0.5$; Max clusters $=500$. 


\section{$483 \underline{\text { References }}$}

484 Angelstam, P. (2004) Habitat thresholds and effects of forest landscape change on the distribution and 485 abundance of black grouse and capercaillie. Ecological Bulletins 51: 173-187.

486

487 Atwood J., Fitz, V.L. \& Bamesberger, J.E. (1991) Temporal patterns of singing activity at leks of the 488 white-bellied emerald. The Wilson Bulletin 103(3): 373-386.

489

490 Bardeli, R., Wolff, D., Kurth, F., Koch, M., Tauchert, K.-H. \& Frommolt, K.-H. (2010) Detecting bird sounds in a complex acoustic environment and application to bioacoustic monitoring. Pattern Recognition Letters. 31(12): 1524-1534.

493

Brandes, T.S. (2008). Automated sound recording and analysis techniques for bird surveys and conservation. Bird Conservation International, 18(S1), S163-S173

Briggs, F., Lakshminarayanan, B., Neal, L., Fern, X.Z., Raich, R., Hadley, S.J.K., Hadley, A.S. multi-label approach. Journal of the Acoustical Society of America 131:4640-4650.

Celis-Murillo, A., Deppe, J.L. \& Allen, M.F. (2009) Using soundscape recordings to estimate bird species abundance, richness, and composition. Journal of Field Ornithology, 80: 64-78.

507 Cornec, C., Hingrat, Y. \& Rybak, F. (2014) Individual Signature in a Lekking Species: Visual and

508 Acoustic Courtship Parameters May Help Discriminating Conspecifics in the Houbara Bustard. 509 Ethology, 120: 726-737. 
511 Dawson, D.K. \& Efford, M.G. (2009) Bird population density estimated from acoustic signals.

512 Journal of Applied Ecology, 46: 1201-1209.

513

514 Digby, A., Towsey, M., Bell, B. D. and Teal, P. D. (2013), A practical comparison of manual and 515 autonomous methods for acoustic monitoring. Methods in Ecology and Evolution 4: 675-683.

517 Eaton, M.A., Aebischer, N.J., Brown, A.F., Hearn, R.D., Lock, L., Musgrove, A.J., Noble, D.G., 518 Stroud, D.A. \& Gregory, R.D. (2015) Birds of Conservation Concern 4: the population status 519 of birds in the United Kingdom, Channel Islands and Isle of Man. British Birds 108: 708-746.

Ehnes, M. \& Foote, J.R. (2015) Comparison of autonomous and manual recording methods for discrimination of individually distinctive Ovenbird songs. Bioacoustics 24(2): 111:121.

524 Ewing, S.R., Eaton, M.A, Poole, T.F, Davies, M. \& Haysom, S. (2012) The size of the Scottish 525 population of Capercaillie Tetrao urogallus: results of the fourth national survey. Bird Study $526 \quad 59(2): 126-138$.

Eyre, T.J., Ferguson, D.J., Hourigan, C.L., Smith, G.C., Mathieson, M.T., Kelly, A.L., Venz, M.F., Hogan, L.D. \& Rowland, J. (2014) Terrestrial Vertebrate Fauna Survey Assessment Guidelines for

530 Queensland. Department of Science, Information Technology, Innovation and the Arts, Queensland 531 Government, Brisbane.

533 Gilbert, G., Tyler, G.A. \& Smith, K.W. (2002) Local annual survival of booming male great bittern Botaurus stellaris in Britain, in the period 1990-1999. Ibis 144: 51-61.

536 Hancock, M., Baines, D., Gibbons, D., Etheridge, B., \& Shepherd, M. (1999) Status of male Black 537 Grouse Tetrao tetrix in Britain in 1995-96. Bird Study, 46(1): 1-15 
539 Hobson, K.A., Rempel, R.S., Greenwood, H., Turnbull, B. \& Van Wilgenburg, S.L. (2002) Acoustic

540 Surveys of Birds Using Electronic Recordings: New Potential from an Omnidirectional Microphone

541 System Wildlife Society Bulletin (1973-2006) 30(3): 709-720.

542

543 Haysom, S. (2013) Capercaillie Survey Methods. Scottish Natural Heritage.

545 Hjorth, I. (1970) Reproductive behaviour in Tetraonidae, with special reference to males. Viltrevy 7 :

$546 \quad 184-596$

547

548 Jacob, G., Debrunner, R., Gugerli, F., Schmid B. \& Bollmann, K. (2010) Field surveys of capercaillie

549 (Tetrao urogallus) in the Swiss Alps underestimated local abundance of the species as revealed by

550 genetic analyses of non-invasive samples. Conservation Genetics 11(1): 33-44.

551

552 Laiolo, P. (2010) The emerging significance of bioacoustics in animal species conservation.

553 Biological Conservation, 143(7):1635-1645.

554

555 Laiolo, P., Bañuelos, M.J., Blanco-Fontao, B., García, M. \& Gutiérrez, G. (2011) Mechanisms

556 underlying the bioindicator notion: Spatial association between individual sexual performance and

557 community diversity. PLoS ONE 6(7): e22724.

558

559 Marques, T. A., Thomas, L., Martin, S.W., Mellinger, D.K., Ward, J.A., Moretti, D.J. \& Tyack, P.L.

560 (2013). Estimating animal population density using passive acoustics. Biological Reviews of the

561 Cambridge Philosophical Society, 88(2): 287-309.

562

563 Moss, R. (2001) Second extinction of capercaillie (Tetrao urogallus) in Scotland? Biological

564 Conservation 101(2): 255-257.

565

ScholarOne, 375 Greenbrier Zُ4 Îve, Charlottesville, VA, 22901 
566 Moss, R. \& Lockie, I. (1979) Infrasonic components in the song of the Capercaillie Tetrao urogallus.

567 Ibis 121: 95-97.

568

569 Oppel, S., Hervias, S., Oliveira, N., Pipa, T., Silva, C., Geraldes, P., Goh, M., Immler, E. \& McKown,

570 M. (2014) Estimating population size of a nocturnal burrow-nesting seabird using acoustic monitoring 571 and habitat mapping. Nature Conservation 7: 1-13.

572

573

Peake, T.M. \& McGregor, P.K. (2001) Corncrake Crex crex census estimates: a conservation application of vocal individuality. Animal Biodiversity \& Conservation 24: 81-90.

575

576 Picozzi, N., Catt D.C. \& Moss, R. (1992) Evaluation of Capercaillie Habitat. Journal of Applied 577 Ecology, 29(3): 751-762.

578

579

Pocock, M. J. O., Newson, S. E., Henderson, I. G., Peyton, J., Sutherland, W. J., Noble, D. G., Ball, S.

G., Beckmann, B. C., Biggs, J., Brereton, T., Bullock, D. J., Buckland, S. T., Edwards, M., Eaton, M. A., Harvey, M. C., Hill, M. O., Horlock, M., Hubble, D. S., Julian, A. M., Mackey, E. C., Mann, D. J., Marshall, M. J., Medlock, J. M., O'Mahony, E. M., Pacheco, M., Porter, K., Prentice, S., Procter, D.

A., Roy, H. E., Southway, S. E., Shortall, C. R., Stewart, A. J. A., Wembridge, D. E., Wright, M. A. and Roy, D. B. (2015) Developing and enhancing biodiversity monitoring programmes: a collaborative assessment of priorities. Journal of Applied Ecology 52: 686-695.

R Core Team (2013). R: A language and environment for statistical computing. R Foundation for 588 Statistical Computing, Vienna, Austria. URL http://www.R-project.org/.

591 URL http://www.rstudio.com/. 
593 Sedláček, O., Vokurková, J., Ferenc, M., Djomo, E.N., Albrecht, T., Hořák, D. (2015). A comparison

594 of point counts with a new acoustic sampling method: a case study of a bird community from the

595 montane forests of Mount Cameroon. Ostrich 86: 213-220

596

597 Simons, T.R., Pollock, K.H., Wettroth, J.M., Alldredge, M.W., Pacifici, K. \& Brewster, J. (2009)

598 'Sources of Measurement Error, Misclassification Error, and Bias in Auditory Avian Point Count

599 Data' in David L Thomson, Evan G. Cooch, Michael J. Conroy (eds) Modeling Demographic

$600 \quad$ Processes In Marked Populations Springer

601

602 Storch, I. (1995) Annual home ranges and spacing patterns of Capercaillie in central Europe. Journal 603 of Wildlife Management 59: 392-400.

604

605 Storch, I. (2000) Conservation status and threats to grouse worldwide: an overview. Wildlife

606 Biology 6(4): 195-204.

607

608 Summers, R.W., Dugan, D. \& Proctor, R. (2010) Numbers and breeding success of Capercaillies

609 Tetrao urogallus and Black Grouse T. tetrix at Abernethy Forest, Scotland. Bird Study 57(4): 437-

610446.

611

612 Summers, R.W., Proctor, R., Thorton, M. \& Avey, G. (2004) Habitat selection and diet of the

613 Capercaillie Tetrao urogallus in Abernethy Forest, Strathspey, Scotland. Bird Study 51(1): 58-68.

615 Turgeon, P. J., S. L. Van Wilgenburg, and K. L. Drake. (2017) Microphone variability and

616 degradation: implications for monitoring programs employing autonomous recording units. Avian

617 Conservation and Ecology 12(1):9.

618

619 Venier, L.A., Holmes, S.B., Holborn, G.W., Mcilwrick, K.A., Brown, G., 2012. Evaluation of an 620 automated recording device for monitoring forest birds. Wildlife Society Bulletin 36: 30-39. 
622 Wegge, P., Rolstad, J. \& Storaunet, K.O (2013) On the spatial relationship of males on "exploded"

623 leks: the case of Capercaillie grouse Tetrao urogallus examined by GPS satellite telemetry. Ornis

624 Fennica 90:222-235.

625

626 Wildlife Acoustics (2016) Kaleidoscope 4.0.0 Pro Software.

627

628 Wilkinson, N. (26 July 2017). National capercaillie survey 2015-16.

629 http://www.rspb.org.uk/community/ourwork/b/biodiversity/archive/2017/07/26/national-capercaillie-

630 survey-2015-16.aspx. Accessed 24 October 2017

631

632 Yip, D. A., L. Leston, E. M. Bayne, P. Sólymos, and A. Grover (2017). Experimentally derived 633 detection distances from audio recordings and human observers enable integrated analysis of point 634 count data. Avian Conservation and Ecology 12(1):11.

635

636 Zwart, M.C., Baker, A., McGowan, P.J.K. \& Whittingham, M.J. (2014) The Use of Automated 637 Bioacoustic Recorders to Replace Human Wildlife Surveys: An Example Using Nightjars. PLoS ONE 638 9(7): e102770.

639 
$\underline{\text { Tables }}$

641

642 Table 1. The error matrix produced from: (a) the clustering process which produced

643 the classifier from the single-day training dataset, and (b) applying this classifier to

644 the 13 day analysis dataset. False negatives are where the species was present but

645 not detected by the software (read along the rows less the diagonal cell). False

646 positives are where the software identified the species to be present when it was not

647 (read down the columns less the diagonal cell).

648 (a) Training dataset

\begin{tabular}{ll|ll|ll}
\hline & & \multicolumn{2}{l}{ Software classifier } & \multicolumn{2}{l}{} \\
& & Capercaillie & Other & TOTAL & False negative (\%) \\
\hline Manual & Capercaillie & $\mathbf{5 2}$ & 206 & 258 & 79.8 \\
identification & 28 & $\mathbf{5 , 1 1 5}$ & 5,143 & 0.58 \\
\hline & Other & 80 & 5,321 & 5,401 & \\
& TOTAL & 3.87 & & \\
\hline
\end{tabular}

649

650 (b) Analysis dataset

\begin{tabular}{ll|ll|ll}
\hline & & \multicolumn{2}{|l|}{ Software classifier } & \multicolumn{2}{c}{} \\
& & Capercaillie & Other & TOTAL & False negative (\%) \\
\hline $\begin{array}{l}\text { Manual } \\
\text { identification }\end{array}$ & Capercaillie & $\mathbf{7 5 8}$ & $\begin{array}{l}1,399 \\
\text { (estimate) }\end{array}$ & 2,157 & 64.9 \\
& Other & 149 & $\begin{array}{l}\mathbf{1 1 , 3 2 0} \\
\text { (estimate) }\end{array}$ & 11,469 & 1.3 \\
\hline & TOTAL & 907 & 12,719 & 13,626 & \\
& False positive (\%) & 16.4 & 11.0 & & \\
\hline
\end{tabular}

651

652 
655 Table 2. Total numbers of phrase segments at each recorder location.

656

\begin{tabular}{|c|c|c|c|c|c|}
\hline Recorder & & 9333 & 9898 & 9558 & 9573 \\
\hline Lek site & & $A$ & $A$ & $\mathrm{~B}$ & C \\
\hline Lek count (males) & & 3 & 3 & 7 & 5 \\
\hline \multirow{2}{*}{ All phrase segments } & $\begin{array}{l}\text { Microphone } 1 / 2 \\
\text { Left/Right }\end{array}$ & $449 / 75$ & $1445 / 743$ & $186 / 1750$ & $5599 / 3379$ \\
\hline & Total & 524 & 2188 & 1936 & 8978 \\
\hline \multirow{3}{*}{$\begin{array}{l}\text { Capercaillie phrase } \\
\text { segments }\end{array}$} & $\begin{array}{l}\text { Microphone 1/2 } \\
\text { Left/Right }\end{array}$ & $4 / 0$ & $206 / 59$ & $0 / 152$ & $272 / 65$ \\
\hline & $\begin{array}{l}\text { Total } \\
\text { (\% of all phrases) }\end{array}$ & $4(0.76 \%)$ & $265(12.11 \%)$ & $152(7.85 \%)$ & $337(3.75 \%)$ \\
\hline & Mean(range)/day & $0.31(0-2)$ & $20.38(0-146)$ & $11.69(0-40)$ & $25.92(0-101)$ \\
\hline
\end{tabular}

657

658

659

660

661 
663 Table 3. Spearman's rank correlation of weather conditions with total number of 664 calls per day.

\begin{tabular}{llll}
\hline Variable & $\mathbf{S}$ & $\mathbf{p}$ & rho \\
\hline Wind & 576.64 & 0.036 & -0.584 \\
Temperature & 523.22 & 0.135 & -0.437 \\
Rain & 532.46 & 0.111 & -0.463 \\
\hline
\end{tabular}

665

666 
Figures

668

669 Figure 1. Typical spectrogram of capercaillie call, showing frequency spectrum in upper 670 window and amplitude in lower window.

671

672 Figure 2. Box plot of mean frequency of capercaillie phrase segments at each recorder 673 location. The centreline of each box indicates the median value for all phrase segments at 674 each recorder location. Boxes represent the data between lower and upper quartiles, and the 675 whiskers extend to the most extreme data point which is no more than 1.5 times the 676 interquartile range. Outliers in each population are represented by dots.

678 Figure 3. Total number of capercaillie phrase segments recorded per day, across all detectors. 679

680 Figure 4. Capercaillie vocalizations in relation to sunrise time. Box plots indicate median 681 times, quartiles and ranges for capercaillie phrase segments at each recorder location, in 682 realtion to sunrise. Box plot width indicates relative sample size. The median time for all 683 capercaillie phrase segments recorded is indicated by the dotted vertical line at 36 minutes 684 before sunrise. The kernel density of capercaillie phrase segments over time is shown by the 685 solid line.

687 Figure 5. Timing of vocalizations in relation to date, for all recorder locations combined. The 688 size of circles indicates the number of phrase segments recorded within each 10 minute 689 recording period.

690 
691 Figure 6. Inverse relationship between number of phrase segments recorded per day and wind

692 speed. Spearman's rank correlation coefficient $(S=576.64$, $p$-value $=0.03604$, rho $=-$

$693 \quad 0.5841723)$.

694

695 


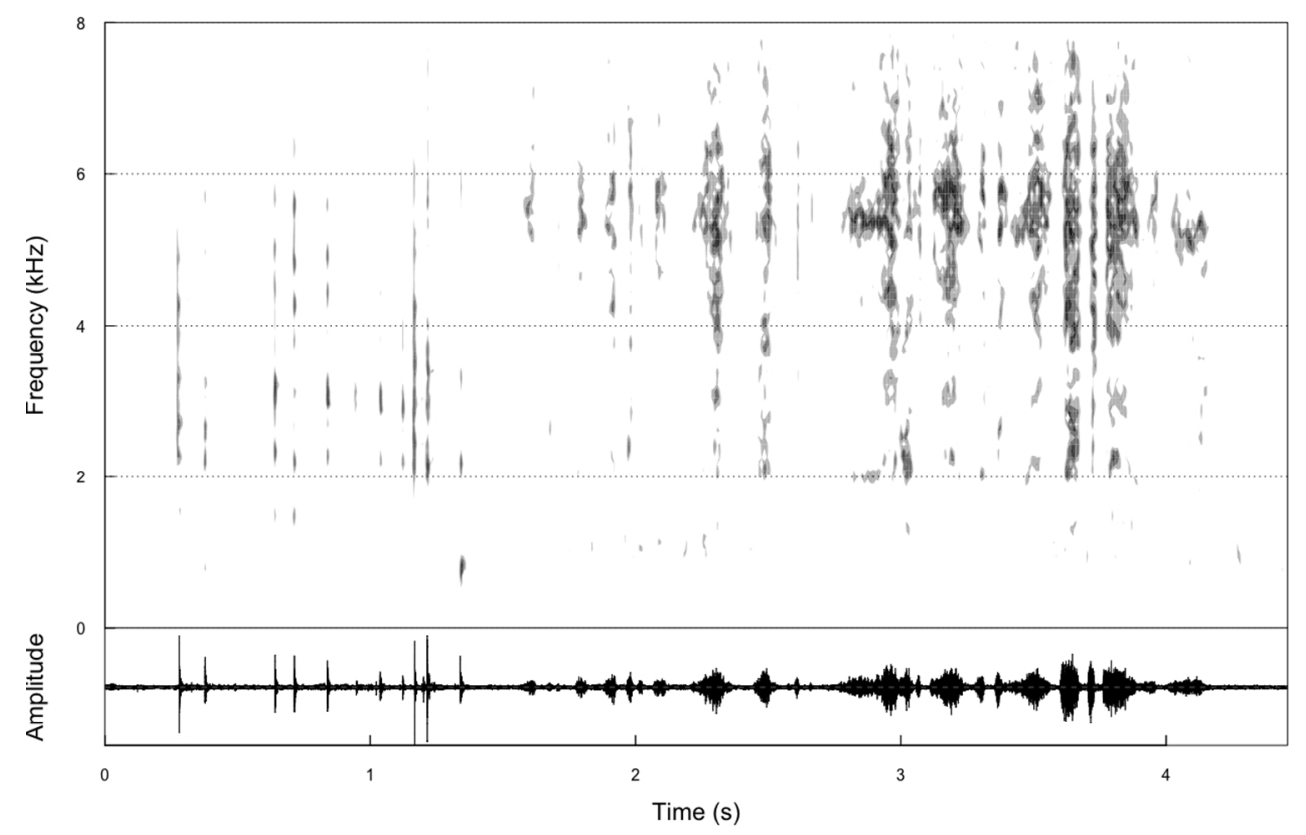

Figure 1. Typical spectrogram of capercaillie call, showing frequency spectrum in upper window and amplitude in lower window.

$456 \times 302 \mathrm{~mm}(72 \times 72 \mathrm{DPI})$ 


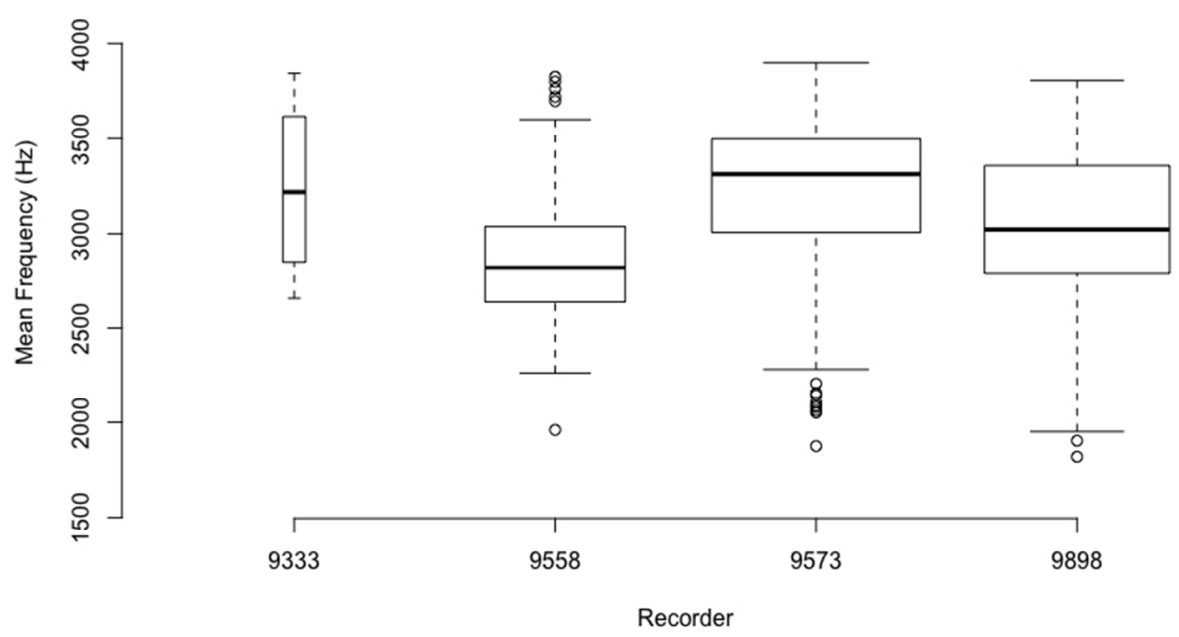

Figure 2. Box plot of mean frequency of capercaillie phrase segments at each recorder location. The centreline of each box indicates the median value for all phrase segments at each recorder location. Boxes represent the data between lower and upper quartiles, and the whiskers extend to the most extreme data point which is no more than 1.5 times the interquartile range. Outliers in each population are represented by dots. Box plot width indicates relative sample size.

$315 \times 191 \mathrm{~mm}(72 \times 72 \mathrm{DPI})$ 


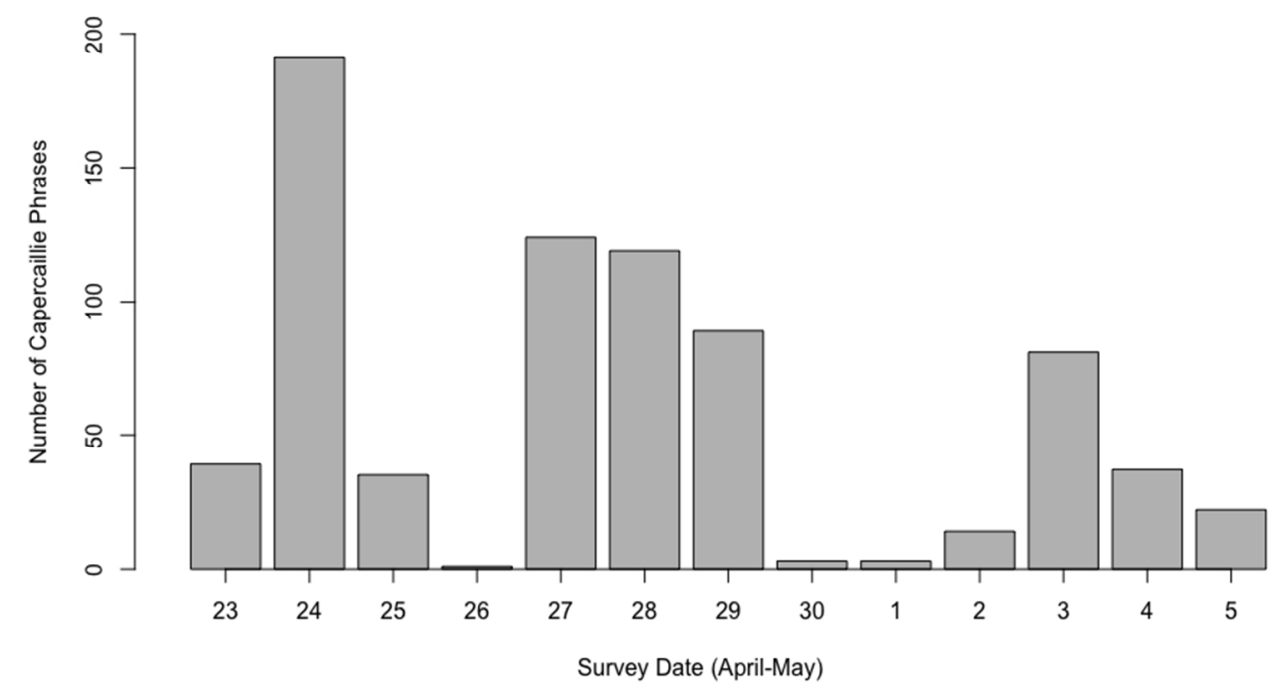

Figure 3. Total number of capercaillie phrase segments recorded per day, across all detectors. $318 \times 193 \mathrm{~mm}(72 \times 72 \mathrm{DPI})$ 


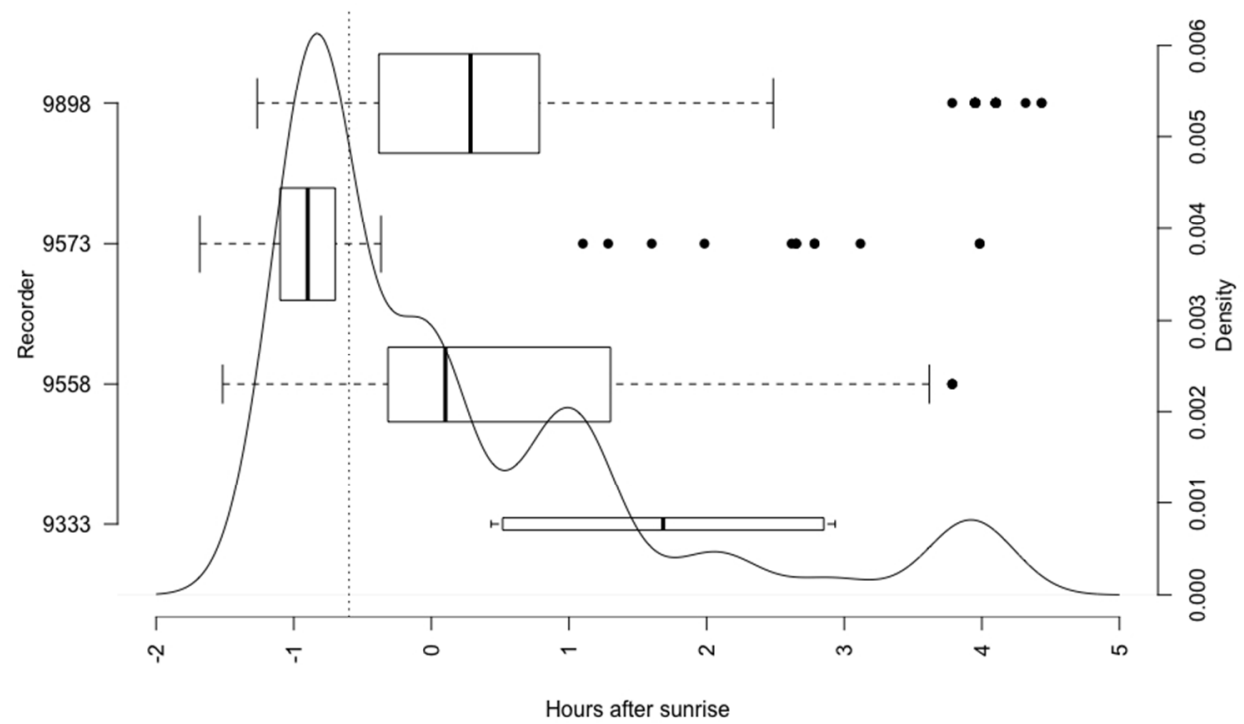

Figure 4. Capercaillie vocalizations in relation to sunrise time. Box plots indicate median times, quartiles and ranges for capercaillie phrase segments at each recorder location, in relation to sunrise. Box plot width indicates relative sample size. The median time for all capercaillie phrase segments recorded is indicated by the dotted vertical line at 36 minutes before sunrise. The kernel density of capercaillie phrase segments over time is shown by the solid line.

$336 \times 204 \mathrm{~mm}(72 \times 72$ DPI $)$ 


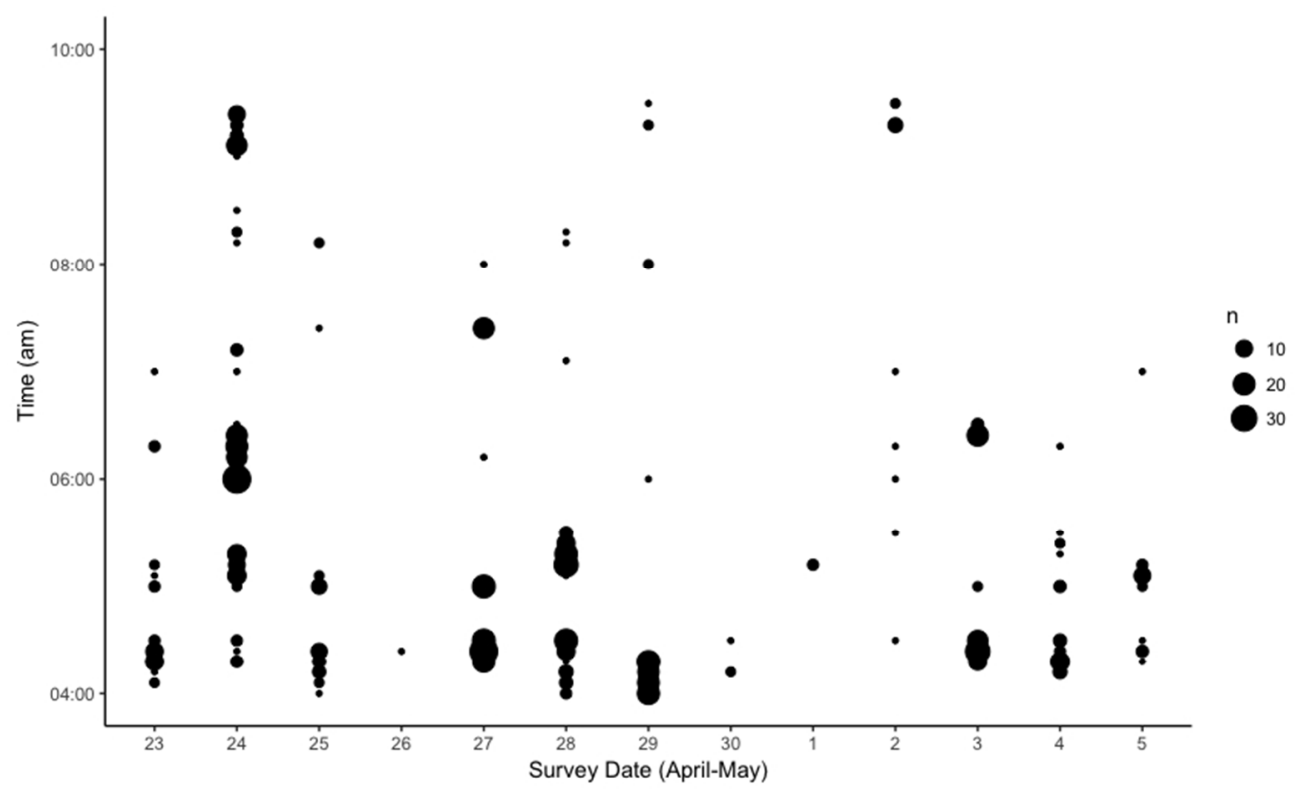

Figure 5. Timing of vocalizations in relation to date, for all recorder locations combined. The size of circles indicates the number of phrase segments recorded within each 10 minute recording period.

$$
299 \times 181 \mathrm{~mm}(72 \times 72 \mathrm{DPI})
$$




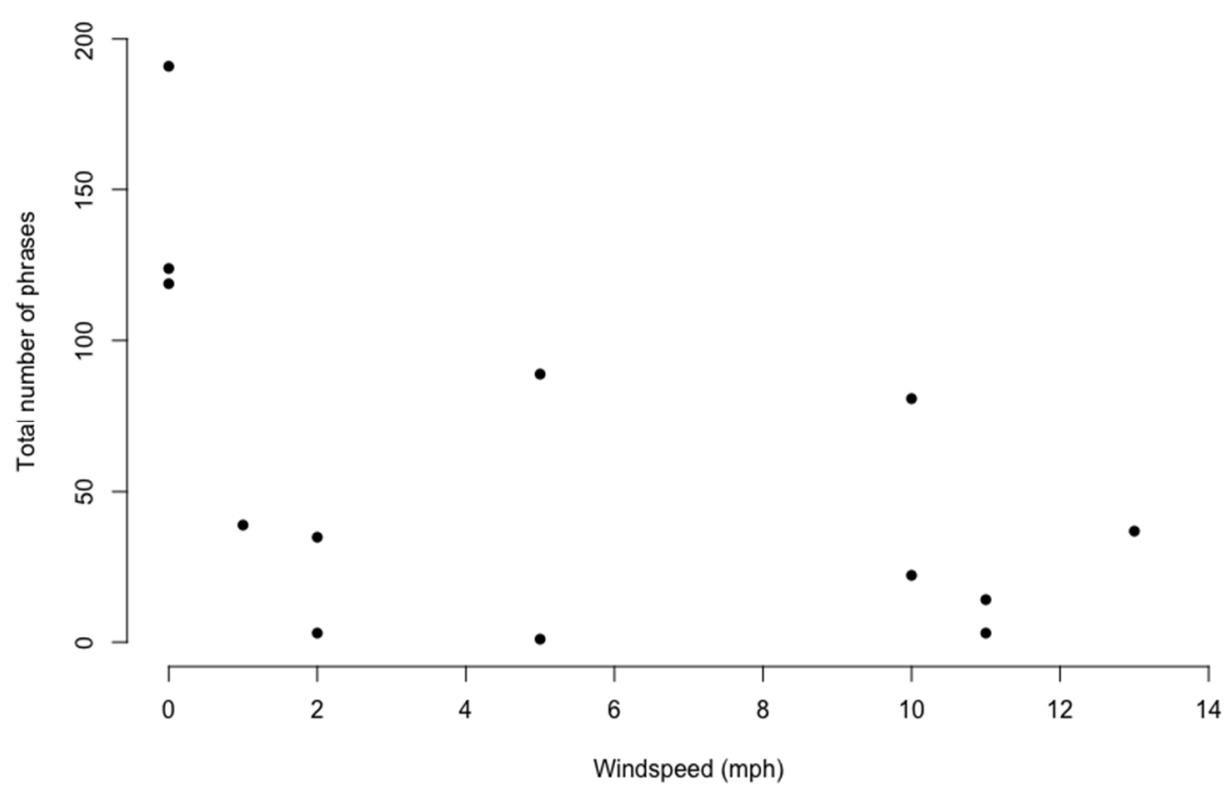

Figure 6. Inverse relationship between number of phrase segments recorded per day and wind speed. Spearman's rank correlation coefficient $(S=576.64, p$-value $=0.036$, rho $=-0.584)$.! + !n +

$304 \times 214 \mathrm{~mm}(72 \times 72$ DPI $)$ 
Table 1. The error matrix produced from: (a) the clustering process which produced the classifier from the single-day training dataset, and (b) applying this classifier to the 13 day analysis dataset. False negatives are where the species was present but not detected by the software (read along the rows less the diagonal cell). False positives are where the software identified the species to be present when it was not (read down the columns less the diagonal cell).

(a) Training dataset

\begin{tabular}{ll|ll|ll}
\hline & & \multicolumn{2}{l}{ Software classifier } & \multicolumn{2}{l}{} \\
& & Capercaillie & Other & TOTAL & False negative (\%) \\
\hline $\begin{array}{l}\text { Manual } \\
\text { identification }\end{array}$ & Capercaillie & $\mathbf{5 2}$ & 206 & 258 & 79.8 \\
& Other & 28 & $\mathbf{5 , 1 1 5}$ & 5,143 & 0.58 \\
\hline & TOTAL & 80 & 5,321 & 5,401 & \\
& False positive (\%) & 35.0 & 3.87 & & \\
\hline
\end{tabular}

(b) Analysis dataset

\begin{tabular}{ll|ll|ll}
\hline & & \multicolumn{2}{|l|}{ Software classifier } & \multicolumn{2}{c}{} \\
& & Capercaillie & Other & TOTAL & False negative (\%) \\
\hline $\begin{array}{llll}\text { Manual } \\
\text { identification }\end{array}$ & Capercaillie & $\mathbf{7 5 8}$ & $\begin{array}{l}1,399 \\
(\text { estimate) }\end{array}$ & 2,157 & 64.9 \\
& Other & 149 & $\begin{array}{l}\mathbf{1 1 , 3 2 0} \\
(\text { estimate) }\end{array}$ & 11,469 & 1.3 \\
\hline & TOTAL & 907 & 12,719 & 13,626 & \\
& False positive (\%) & 16.4 & 11.0 & & \\
\hline
\end{tabular}


Table 2. Total numbers of phrase segments at each recorder location.

\begin{tabular}{|c|c|c|c|c|c|}
\hline Recorder & & 9333 & 9898 & 9558 & 9573 \\
\hline Lek site & & A & A & B & C \\
\hline Lek count (males) & & 3 & 3 & 7 & 5 \\
\hline \multirow{2}{*}{ All phrase segments } & $\begin{array}{l}\text { Microphone 1/2 } \\
\text { Left/Right }\end{array}$ & $449 / 75$ & $1445 / 743$ & $186 / 1750$ & $5599 / 3379$ \\
\hline & Total & 524 & 2188 & 1936 & 8978 \\
\hline \multirow{3}{*}{$\begin{array}{l}\text { Capercaillie phrase } \\
\text { segments }\end{array}$} & $\begin{array}{l}\text { Microphone 1/2 } \\
\text { Left/Right }\end{array}$ & $4 / 0$ & $206 / 59$ & $0 / 152$ & $272 / 65$ \\
\hline & $\begin{array}{l}\text { Total } \\
\text { (\% of all phrases) }\end{array}$ & $4(0.76 \%)$ & $265(12.11 \%)$ & $152(7.85 \%)$ & $337(3.75 \%)$ \\
\hline & Mean(range)/day & $0.31(0-2)$ & $20.38(0-146)$ & $11.69(0-40)$ & $25.92(0-101)$ \\
\hline
\end{tabular}


Table 3. Spearman's rank correlation of weather conditions with total number of calls per day.

\begin{tabular}{llll}
\hline Variable & $\mathbf{S}$ & $\mathbf{p}$ & rho \\
\hline Wind & 576.64 & 0.036 & -0.584 \\
Temperature & 523.22 & 0.135 & -0.437 \\
Rain & 532.46 & 0.111 & -0.463 \\
\hline
\end{tabular}

